\title{
High methoxyl pectin from dragon fruit (Hylocereus polyrhizus) peel
}

\begin{abstract}
Pectin from different fractions of dragon fruit (Hylocereus polyrhizus) peel was extracted using $1 \%$ citric acid and the physico-chemical characteristics of the pectin were studied. The highest pectin yield ( $26.38 \%$ on dry weight basis) was obtained from fresh inner layer of the peel when extraction was carried out at temperature: $73{ }^{\circ} \mathrm{C}$, time: $67 \mathrm{~min}, \mathrm{pH}: 2.03$, and sample to citric acid ratio: 1:4 (w/v). The pectin also demonstrated the highest degree of esterification (63.74\%) when compared with pectin from other fractions of the dragon fruit peel investigated in this study. The calculated degree of esterification confirmed that the extracted pectin is a high methoxyl pectin. The molecular weight of the pectin determined using size exclusion chromatography was $0.88 \times 105$ Da. Monosaccharide composition determined using high performance liquid chromatography revealed that the pectin was predominantly constituted of galacturonic acid (39.11\%), followed by moderate concentrations of mannose, rhamnose, galactose, glucose and minor amounts of xylose and arabinose. The pectin exhibited Newtonian behaviour at concentrations of $0.5 \%$ and $1.0 \%$, and pseudoplastic behaviour at concentrations of $2.0 \%$ and $3.0 \%$. Although the viscosity of the dragon fruit peel pectin was lower than that of commercial apple and citrus pectins, it can be used as a functional and health ingredient in low viscous foods and beverages.
\end{abstract}

Keyword: Extraction; Physico-chemical; Characterisation; Dragon fruit peel; High methoxyl pectin 